\title{
ABORDAGEM DA DOR GÊNITO-PÉLVICA/PENETRAÇÃO
}

\author{
Júlia Kefalás Troncon'; Heliana Aparecida da Silva Pandochi²; Lúcia Alves Lara ${ }^{3}$ \\ APPROACH TOWARDS GENITO-PELVIC PAIN/PENETRATION DISORDER
}

Resumo: A dor durante a relação sexual com penetração vaginal pode ser caracterizada como uma disfunção sexual e tem prevalência elevada em mulheres. Pode ter causas orgânicas e psíquicas associadas, por isso sua abordagem deve ser multidisciplinar e envolver médicos, psicólogos e fisioterapeutas. O presente artigo visa criar um protocolo de abordagem dessa disfunção, facilitando seu diagnóstico e tratamento.

Palavras-chave: dispareunia; vaginismo; vulvodínia.

\begin{abstract}
Pain during sexual intercourse can be characterized as Genito-Pelvic Pain/Penetration Disorder, and has elevated prevalence among women. It may have both organic and psychological causes, and for that reason should be approached by a multidisciplinary team involving medical doctors, psychologists and physical therapists. This paper aims to create a protocol for the diagnosis and treatment of this disorder. Keywords: dyspareunia; vaginismus; vulvodynia
\end{abstract}

\footnotetext{
1 Ginecologista, com Mestrado pela USP, médica assistente do Ambulatório de Estudos em Sexualidade Humana, Faculdade de Medicina de Ribeirão Preto, USP, e membro da Comissão Nacional Especializada (CNE) em sexologia da Federação Brasileira de Ginecologia e Obstetrícia (FEBRASGO). E-mail: juktroncon@yahoo.com.br

${ }^{2}$ Fisioterapeuta, com Mestrado pela USP em tratamento da dor gênito-pélvica de penetração. E-mail: honaili@hotmail.com

${ }^{3}$ Ginecologista, médica responsável do Ambulatório de Estudos em Sexualidade Humana, Faculdade de Medicina de Ribeirão Preto, USP, e presidente da Comissão Nacional Especializada (CNE) em sexologia da Federação Brasileira de Ginecologia e Obstetrícia (FEBRASGO). E-mail: luciaalvess@yahoo.com.br
} 


\section{Introdução}

O Transtorno de Dor Gênito-Pélvica/Penetração baseia-se na dificuldade persistente ou recorrente de alcançar a penetração vaginal durante o coito, ou dor vulvovaginal ou pélvica durante relação sexual ou tentativa de penetração vaginal. Caracteriza-se também pela presença de medo ou ansiedade relacionado à dor vulvovaginal ou pélvica em antecipação, durante ou como resultado de penetração vaginal, além da presença de tensão ou endurecimento dos músculos do assoalho pélvico durante a tentativa de penetração vaginal. 0 período de persistência dos sintomas precisa ser maior que seis meses, o sofrimento do indivíduo e a exclusão de transtornos mentais, violência sexual pelo parceiro ou outros fatores estressores que possam resultar em dor sexual também devem ser considerados para estabelecimento do diagnóstico (APA, 2013; LAHAIE et al., 2015).

Com relação os transtornos álgicos pertinentes à vulva, há dois grandes subgrupos: dor vulvar, que se refere à situações com causa orgânica estabelecida, e vulvodínia. A vulvodínia é definida como dor vulvar por mais de três meses e sem etiologia identificável, embora se saiba que pode ter alguns fatores envolvidos em sua origem como os musculoesqueléticos, neuroproliferativos e psicossomáticos. Quanto à dor vulvar, pode ser de origem infecciosa (herpes genital, candidíase), inflamatória (como líquen), neoplásica, iatrogênica (pós radioterapia, por exemplo), hormonal, entre outras (BORNSTEIN et al, 2016).

Estimativa americana revelou que aproximadamente de $15 \%$ a $20 \%$ das mulheres com idades entre 18 e 64 anos são acometidas por essa disfunção, com cerca de 6 milhões de muIheres apresentando dispareunia (WHITMORE et al., 2007). Uma pesquisa brasileira evidenciou a prevalência de $17,8 \%$ para esta condição (ABDO, 2004).

Para facilitar o diagnóstico e assim uma abordagem otimizada, serão ilustrados em uma divisão didática a seguir a dispareunia, o vaginismo e a vulvodínia.

\section{Dispareunia}

Pode ser classificada como superficial ou profunda e é definida como a presença de dor genital durante a relação sexual, podendo es- tar associada a fatores físicos e/ou psicológicos (MORRIS et al., 2006). A dispareunia superficial refere-se à dor percebida em região vulvovestibular, no início da penetração, ou com o movimento do pênis dentro da vagina, durante a relação sexual, e pode ter múltiplas etiologias, principalmente relacionadas com patologias genitais ou pélvicas como: atrofia genital na pós-menopausa, ressecamento vaginal e lubrificação vaginal inadequada, infecção do trato urinário, prolapso, líquen, entre outros (BINIK, 2010). Já a dispareunia de profundidade, na qual a dor se manifesta no fundo vaginal e hipogástrio, está frequentemente associada a um padrão de dor que se reproduz em outras situações fora do ato sexual e dor pélvica crônica propriamente dita, tendo causa predominantemente orgânica. Pode ser persistente, quando a dor ocorre em todas as relações, ou condicional, quando a dor se apresenta em algumas posições, tipos de estimulações ou relacionada a um parceiro específico (HEIM et al., 2001; MORRIS et al., 2006).

\section{Vaginismo}

Com a publicação do Manual Diagnóstico e Estatístico de Transtornos Mentais $\mathrm{V}$ (DSM V), sua definição se tornou mais abrangente, tendo sido englobada pelo conceito de desordem de dor gênito-pélvica, e passando a ser caracterizada como a dificuldade na penetração vaginal associada à dor, ao medo e à contração da musculatura do assoalho pélvico no momento da relação sexual (APA, 2013; LAHAIE et al., 2015).

\section{Vulvodínia}

Vuvlodínia é a dor vulvar idiopática de pelo menos três meses de duração (PUKALL et al., 2016). Caracteriza-se como desconforto vulvar, comumente referido como dor tipo queimação em vulva e vestíbulo vaginal, e deverá estar associada à ausência de patologias infecciosas, inflamatórias, neoplásicas ou neurológicas (HAEFNER et al., 2005). A fisiopatologia da vulvodínia é ainda incerta e muito provavelmente multifatorial, englobando neuroproliferação, dor miofascial, hormonais e psicossociais, redução de limiar de dor, polimorfismos genéticos, entre outros (PUKALL et al., 2016). Pode ser classificada em espontânea ou provocada (por contato, relação sexual), e 
em generalizada ou localizada (vestibulodínia, clitorodínia, hemivulvodínia) (BORNSTEINET, 2016). Entre os fatores hormonais, o hipoestrogenismo associado ao estado menopausal, e que pode estar associado à síndrome genito-urinária da menopausa, tem importante papel já que pode levar ao aumento de densidade de terminações nervosas na vulva e vagina (STRAUB, 2007).

\section{Diagnóstico}

É necessária uma abordagem detalhada a fim de determinar os fatores predisponentes e determinantes para ocorrência desta disfunção (Quadro 1), a qual deve ser feita preferencialmente pelo médico ginecologista.

Nos casos de dispareunia e vaginismo, a inspeção e o toque vaginal devem ser minuciosos, enquanto procura-se observar a presença de alterações na mucosa, espasmo muscular, bem como de pontos de gatilho, que são áreas focais de hiperalgesia, localizadas na musculatura perineal superficial. Embora de fisiopatologia ainda incerta, trauma na parede vaginal e cirurgias prévias podem ser fatores desencadeantes (ALVAREZ et al., 2002).

O diagnóstico de vaginismo pode ser aventado pelo relato de dificuldade ou impossibilidade da penetração vaginal, associado à dor e ao medo, e será complementado necessariamente pelo exame físico, onde comumente é observada uma postura defensiva manifestada pela adução dos membros inferiores na tentativa de esquivar-se do contato das mãos do examinador no momento do exame e contração involuntária dos músculos do assoalho pélvico (LAHAIE et al., 2015).

O diagnóstico de vulvodínia é auxiliado pelo teste do cotonete, que consiste em utilizar um swab ou cotonete para realizar leve pressão sobre diferentes áreas da vulva, avaliando a manifestação de dor ou desconforto da paciente (HAEFNER et al., 2005). Este teste, contudo, tem ampla variação interobservador e é pouco reprodutível; assim, a anamnese e o exame físico completos são fundamentais para estabelecer o diagnóstico de dor vulvar e afastar outras causas de dor genital (GOLDSTEIN et al., 2016).

Fatores psicogênicos também podem interferir, pois o medo de que a sensação dolorosa volte a aparecer pode fazer com que a mulher apresente postura de defesa, contrain- do involuntariamente os músculos do assoaIho pélvico, levando à persistência do estado de tensão e dor na relação sexual (SCAFURI et al., 2009).

Os distúrbios de excitação com lubrificação inadequada que podem levar à dor coital ou outros distúrbios sexuais, com comprometimento da resposta sexual, serão determinados mediante anamnese adequada, exame físico, exames laboratoriais e de imagem pertinentes a cada caso.

A avaliação da dor gênito-pélvica/penetração pode demandar equipe multiprofissional, mas o papel do médico ginecologista é primordial para identificação da queixa inicial com levantamento do histórico clínico e história sexual pregressa e avaliação das possíveis causas orgânicas e psicossociais relacionadas à queixa. Nos casos em que são identificadas alterações musculoesqueléticas, recomenda-se a avaliação de um profissional fisioterapeuta, a fim de verificar o tônus e a função dos músculos do assoalho pélvico, a existência e localização de pontos dolorosos, bem como a compreensão de contração e repouso voluntário dos músculos do assoalho pélvico pela mulher.

\section{Tratamento}

É importante destacar que a conduta terapêutica relacionada à desordem de dor gênito pélvica/penetração deve ser individualizada, pois depende das condições clinicas, dos achados da avaliação, bem como dos fatores que predispõem à dor. É possível citar o uso do bloqueio loco-regional, realizado quando um ponto de gatilho é identificado e encontra-se muito sensibilizado, impossibilitando assim a realização da massagem intravaginal. Consiste de uma injeção com anestésico local. Realiza-se a injeção de $2 \mathrm{~mL}$ de volume de lidocaina a $1 \%$ sem epinefrina ou procaína a $1 \%$ em cada ponto, semanalmente, por quatro a seis semanas; em casos refratários pode ser utilizada a toxina botulínica (FAGEEH, 2011).

Em relação à vulvodínia, medidas de higiene e cuidado genital adequado com sabão com pH entre 3,5 e 5,5, uso de roupas íntimas de algodão, afastar irritantes vulvares como desodorantes e cremes perfumados, que geralmente contêm álcool, são indispensáveis. Lubrificantes à base de propilenoglicol devem ser substituídos por glicerina ou óleos vegetais. $\mathrm{O}$ uso de anestésicos tópicos como a lidocaína 
mostrou-se benéfico para a dessensibilização dolorosa vulvar (HAEFNER et al., 2005), permitindo em alguns casos o retorno à relação sexual, embora não seja indicada como terapia de longo prazo (GOLDSTEIN et al., 2016).

Dado o caráter multifatorial da vulvodínia, algumas recomendações para o tratamento abrangem também a psicoterapia e a fisioterapia do assoalho pélvico. Acapsaicina, assim como a toxina botulínica, podem ser tratamentos de segunda linha quando os demais falharam. A terapia hormonal e acupuntura parecem ser promissoras, mas ainda faltam estudos controlados sobre este tema (GOLDSTEIN et al., 2016).

Os antidepressivos tricíclicos, como a amitriptilina e a nortriptilina, e inibidores seletivos de recaptação de serotonina têm benefício na redução de limiar doloroso. É fundamental alertar a paciente de que o início da resposta demora em média pelo menos quatro semanas. Anticonvulsivantes como a gabapentina e a carbamazepina também são ferramentas terapêuticas possíveis (HAEFNER et al., 2005).

Recomenda-se utilizar uma escala de dor como a escala visual analógica (EVA) previamente ao tratamento, bem como em seu término para avaliar a melhora da dor.

\section{Tratamento fisioterapêutico}

O tratamento fisioterapêutico visa reestabelecer a função dos músculos do assoalho pélvico. As intervenções específicas constam de: i) técnicas de autorrelaxamento e controle da ansiedade que consistem em melhora da consciência corporal e técnicas de controle da respiração, (FAVORÊTO, 2001), ii) dessensibilização: têm como objetivo favorecer o autoconhecimento, já que a mulher é estimulada a visualizar e identificar o clitóris, grandes lábios, pequenos lábios e intróito vaginal através de um espelho e a tocar estas regiões (OLIVEIRA, 2001), iii) alongamento passivo dos músculos adutores da coxa realizado dentro da amplitude normal de movimento com intensidade leve e tempo prolongado (sessenta segundos) bilateral (KISNER et al., 1998), iv) massagem perineal ou massagem intravaginal, que é realizada com a mulher em posição ginecológica, com objetivo de relaxar os músculos do assoalho pélvico, melhorar a circulação sanguínea local e desativar pontos gatilho (SCA-
FURI et al., 2009).

A massagem intravaginal é realizada, como descrita por Oyama et al. (2004), realizando o alongamento muscular da origem para inserção dos músculos do assoalho pélvico (MAPs), no sentido das fibras musculares, com pressão tolerável por cinco minutos do lado direito e cinco minutos do lado esquerdo. Essa técnica se mostra benéfica para reabilitação desses músculos, além de ser um procedimento de baixo custo, não oferecer nenhum risco às mulheres e poder ser realizada pelo parceiro após orientação do fisioterapeuta (MONTENEGRO et al., 2010; SILVA et al., 2016).

A mulher também é orientada a realizar os exercícios para melhora da função dos músculos do assoalho pélvico em seu domicílio. Esses exercícios são compostos por: dez contrações sustentadas dos MAPs por seis segundos, com doze segundos de repouso entre uma contração e outra (fortalecimento de fibras musculares lentas), seguido por dez contrações rápidas de aproximadamente um segundo (para recrutar as fibras musculares rápidas). Deverão ser repetidos três vezes e realizados iniciando na posição supina, evoluindo para posição sentada e depois em posição ortostática (KEGEL,1948; MØRKVED et al., 2003). Cabe ressaltar que esses são exercícios básicos, pois a prescrição exata do número de repetições e repouso dependerá dos achados da avaliação física.

As mulheres não devem interromper as relações sexuais durante o tratamento, pois seu desempenho, bem como dificuldades apresentadas, servem de parâmetros para a evolução do quadro, bem como para nortear a conduta terapêutica.

Outros recursos fisioterapêuticos também podem ser empregados como uso da eletroterapia e biofeedback caso necessário.

O tratamento fisioterapêutico, bem como orientações relacionadas com a reabilitação do assoalho pélvico, deve ser realizado somente por um profissional habilitado.

Quando na avaliação é verificada a presença de pontos gatilhos (trigger-points), que são pontos dolorosos resultantes de espasmo muscular crônico e eles estiverem hipersensibilizados, impossibilitando a massagem, é solicitada avaliação do médico ginecologista com o objetivo de verificar a possibilidade de dessensibilizar aquele ponto através do bloqueio loco 
regional com anestésico, até que a mulher esteja apta a receber a massagem.

\section{Referências}

ABDO C. H. N. Estudo da vida sexual do brasileiro. São Paulo: Bregantini, 2004.

ALVAREZ, D. J.; ROCKWELL, P. G. Trigger points: diagnosis and management. Am fam physician, v. 15, n. 65, p. 653-660, 2002.

American Psychiatric Association (APA). Diagnostic and statistical manual of mental disorders. American Psychiatric Association, 2013.

BINIK, Y. M. The DSM diagnostic criteria for dyspareunia. Arch sex behav. v. 39, n. 2, p. 292-303, 2010.

BORNSTEIN, J.; GOLDSTEIN, A.T.; STOCKDALE, C.K.; BERGERON, S.; PUKALL, C.; ZOLNOUN, D.; COADY, D. 2015 ISSVD, ISSWSH and IPPS Consensus Terminology and Classification of Persistent Vulvar Pain and Vulvodynia. J Sex Med, v. 13, p. 607-612, 2016.

FAGEEH, W. M. Different treatment modalities for refractory vaginismus in western Saudi Arabia. J Sex Med. v. 8, n. 6, p. 1735-1739, 2011.

FAVORÊTO, A. Técnicas gerais utilizadas na terapia sexual: Relaxamento. In: FAVORÊTO, A.; Instituto Paulista de Sexualidade (org.). Aprimorando a saúde sexual. 2. ed. São Paulo: Summus Editorial, p. 99-108, 2001.

GOLDSTEIN, A. T. et al. Pukall Vulvodynia: assessment and treatment. J sex med, v. 13, n. 4, p. 572-590, 2016.

HAEFNER, H. K; COLLINS, M.E.; DAVIS, G.D.; EDWARDS, L.; FOSTER, D.C.; HARTMANN, E.D; KAUFMAN, R.H.; LYNCH, P.J.; MARGESSON, L.J.; MOYAL-BARRACCO, M.; PIPER, C.K.; REED, B.D.; ATEWART, E.G.; WILKISON, E.J. The vulvodynia guideline. J low genit tract dis, v. 9, n. 1, p. 40-51, 2005.

HEIM, L. J.; USAF, M. C.; Evolution and differential diagnosis of dyspareunia. Am fam physician, v. 63, n. 8, p. 1535-1544, 2001.

KEGEL, A. H. Progressive exercise in the func- tional restoration of the perineal muscles. $A m$ J Obstet Gynecol, v. 56, p 238-248, 1948.

KISNER, C.; COLBY, L.A. Exercícios terapêuticos: fundamentos e técnicas. 3. ed. São Paulo: Manole, 1998.

LAHAIE, M. A.; AMSEL, R.; KHALIFÉ, S.; BOYER, S.; FAABORG-ANDERSEN, M.; BINIK, Y.M.Can fear, pain, and muscle tension discriminate vaginismus from dyspareunia/provoked vestibulodynia?: implications for the new DSM5 diagnosis of genito-pelvic pain/penetration disorder. Arch sex behav. v. 44, n. 6, p. 15371550, 2015.

MONTENEGRO, M. L. L. S.; MATEUS-VASCONCELOS, E.C.; CANDIDO DOS REIS, F.J.; ROSA E SILVA, J.C.; NOGUEIRA, A.A.; POLI NETO, O.B. Thiele massage as a therapeutic option for women with chronic pelvic pain caused by tenderness of pelvic floor muscles. JEvalClinPract, 2010.

MORRIS, D. E.; MUKHOPADHYAY, S. Dyspareunia in gynecological practice. Curr Obstet Gynaecol, v. 16, p. 226-233, 2006.

MØRKVED, S.; Bo, K.; SCHEI, B.; SALVESEN, K.A. Pelvic floor muscletraining during pregnancy to prevent urinary incontinence: asingle-blind randomized controlled trial. Obstet Gynecol, v. 101, p. 313-319, 2003.

OYAMA, I. A.; REJBA, A.; LUKBAN, J.C.; FLETCHER， E.; KELLOGG-SPADT， S.; HOLZBERG, A.S.; WHITMORE, K.E. Modified thiele massage as therapeutic intervention for female patients with interstitial cystitis and high-tone pelvic floor dysfunction. Urology. v. 64, n. 5, p. 862865, 2004.

OLIVEIRA, J. S. Vaginismo. In: OLIVEIRA, J. S.; Instituto Paulista de Sexualidade (org.). Aprimorando a saúde sexual. 2. ed. São Paulo: Summus editorial, 2001, p. 291-297.

PUKALL, C. F.; GOLDSTEIN, A.T.; BERGERON, S.; FOSTER, D.; STEIN, A.; KELLOGG-SPADT, S. Vulvodynia: definition, prevalence, impact, and pathophysiological factors. J Sex Med. v. 13, n. 3, p. 291-304, 2016.

SCAFURI A. G.; FERREIRA, B.R.S.; CASTRO FI- 
LHO, J.E.; MIRANDA, E.P.; SOUSA, N.C. Síndrome da bexiga dolorosa cistite intersticial. In: PALMA, P. C. R. Urofisioterapia: aplicações clínicas das técnicas fisioterapêuticas nas disfunções miccionais e do assoalho pélvico. Campinas: Personal Link Comunicações, 2009, p. 429-439.

SILVA, A.P.; MONTENEGRO, M.L.; GURIAN, M.B.; LARA, L.A.; POLI-NETO, O.B.; ROSA E SILVA, J.C. Perineal Massage Improves the Dyspareunia Caused by Tenderness of the Pelvic Floor Muscles. Rev Bras Ginecol Obstret. 2016.
STRAUB, R. H. The complex role of estrogens in inflammation. Endocr Ver, v. 28, n. 5, p. 521574, 2007.

WHITMORE, K.; SIEGGEL, J. F.; KELLOGG-SPADT, S. Intersticial cystitis painful blader syndrome as a cause of sexual pain in women: a diagnosis to consider. J Sex Med, v. 4, n. 3, p. 720-727, 2007.

\section{ANEXO}

Quadro 1: Alguns fatores relacionados à dor gênito-pélvica/penetração (Extraído de manual da febrasgo: Tópicos em saúde sexual)

\section{Causas}

Anatômicas

Infecciosas

Hormonais

Sexuais

Musculares

Patologias diversas

\section{Condições clínicas}

Malformações genitais, septos, cicatrizes cirúrgicas, prolapso genital

Vulvovaginites, infecções sexualmente transmissíveis/doença inflamatória pélvica, cistite intersticial, infecção do trato urinário, úlceras, herpes genital

Ressecamento e atrofia da mucosa vaginal devido à hipoestrogenismo/síndrome gênito-urinária da menopausa, anticoncepcionais hormonais

Disfunção da excitação, desejo sexual hipoativo, anorgasmia, dificuldade de entrega, disfunção sexual do parceiro, deformidade do pênis (doença de Peyronie)

Espasmo muscular, hiperatividade muscular (vaginismo), pontos de gatilho

Endometriose, vulvodínia, liquen, neoplasias 